\title{
Effects of virtual reality cognitive rehabilitation program on cognitive function, physical function and depression in the elders with dementia
}

\begin{abstract}
The aim of this study is to examine the effects of virtual reality cognitive rehabilitation program on cognitive function, physical function and depression of long-term care insurance nongrading elderly dementia using Daytime protection service. For achieve this purpose, 30 dementia elderly were randomly assigned in to the experimental group( $n=15)$ and control group $(n=15)$. All subjects performed a general therapeutic exercise and $20-$ 30 minutes takes virtual reality cognitive rehabilitation program were conducted with experimental group 2 times a week for 8 weeks. The intervention effects were measured by using cognitive function is mini-mental states examination-Korean version(MMSE-K), visual perception is MVPT(Motor-Free Visual Perception Test), Berg's balance scales(BBS), depression test GDS-K and lower limb strength. The results of study represented that the training group showed significant improvement in MMSE$\mathrm{K}(p<.05)$, visual perception(p〈.05), balance(p〈.05), lower limb strength(p〈.05) and depression ( $p<.05)$. In conclusion, the virtual reality cognitive rehabilitation training using improves visual perception included cognitive function, physical function included balance, and lower limb strength and depression. These results suggest that virtual reality training using virtual reality cognitive rehabilitation program is feasible and suitable for mild dementia.
\end{abstract}

Key words: Cognitive Function; Dementia; Virtual Reality
Hyun Ju Moona, Yoo Rim Choib, Sung Kook Lee

a'Kyungpook National University, Taegu; ${ }^{\mathrm{D} T} T a e g u$

Science University, Taegu, Korea

Received : 27 May 2014

Revised : 06 July 2014

Accepted : 09 September 2014

Address for correspondence

Sung-Kook Lee, PH, Ph.D

Department of Preventive Medicine

Kyungpook National University School of Medicine, 80 Daehak-ro, Buk-Gu, Deagu, Korea

Tel: 82-53-420-4860

E-mail: sunglee@knu.ac.kr

\section{INTRODUCTION}

In case of Alzheimer' $\mathrm{s}$ disease(AD), a neurodegenerative disease occupying the largest percentage among the various causes of dementia, it is now possible to ease the symptoms or to delay the progress through early detection and intervention(1). Early Alzheimer dementia patients need to use strategies for efficient storage and retrieval of information in order to overcome the temporal and spatial limitations in their short-term memory. The deterioration of memory observed in early $\mathrm{AD}$ patients is caused by problems in encoding and storing information, namely, defects in information processing functions for learning. Moreover, the retrieval of maintained information can be facilitated by contexts or clues used when the information was stored. In addition to the shrinkage of information, another symptom that begins to appear in early $\mathrm{AD}$ is the decline of attention concentration, which is required for the short-term memory of information coming from the outside. However, the concentration of attention can be activated further when it is accompanied by a positive emotion(2).

Virtual reality is a method of solving problems through applying a video capture system, showing the person' s image on the screen, and interacting with tasks appearing on the screen(3). Virtual reality is largely divided into immersive virtual reality and non-immersive virtual reality(4). In immersive virtual reality, the person wears or connects himself to various kinds of hardware and carries out tasks by implementing signals transmitted through physical activities in the virtual reality. Non-immersive virtual reality is designed to sense body joints through specially invented video captures and express the sensed image in virtual environment on the screen so that the person can interact with the real environment 
in two ways(5). Recently, many studies are being conducted using easily accessible games based on virtual reality, and a therapeutic game means a game using virtual reality for medical purposes(6). In this way, virtual reality is effective for intensive application of various task-oriented interventions necessary for improving physical functions in safe environment(7). However, most of studies on virtual reality programs have been about games for normal people rather than about programs developed for rehabilitation training. Thus, this study purposed to apply "VREHAT," a virtual reality cognitive rehabilitation training system, to dementia elders and to examine the effectiveness of the system by measuring their cognitive function, visual perception, physical functions such as balance ability and lower limb strength, and depression.

\section{METHODS AND METHODS}

\section{Subjects}

The subjects of this study were 30 elders with minor dementia who were excepted from the grades of long-term care insurance and were supported by Daegu City. They were sampled from two day care centers with the guardians' consent, and were divided into a control group $(\mathrm{n}=15)$ who participated in a cognition and rehabilitation program executed by the day care center, and an experimental group $(n=15)$ who participated in an existing cognitive rehabilitation program executed by the day care center and also had virtual reality cognitive rehabilitation training applied for 8 weeks from July to August in 2014, twice a week, and 20-30 minutes per session. The inclusion criteria were: 65-year-old or older elders diagnosed with dementia by a neurologist or a neuropsychiatrist; those who understood the purpose of this study and agreed to participate; and those without paralysis and serious balancing disability. The exclusion criteria were: those with severe dementia who had difficulty in communication; those who were not able to participate in the program due to paralysis or balancing and visual disturbance; and dementia elders with a disease in the lungs, heart, etc. that is inadequate for exercise. Before participating the program, the subjects of the experimental group were given a thorough explanation about the intervention program with a demonstration for being sufficiently familiar with the program.

\section{Intervention Program}

Virtual reality cognitive rehabilitation training system 'VREHAT' consists of motion sensing camera, special motion sensing glove recognizing the hand' $\mathrm{s}$ functional movements, motion sensing glove controller, grip strength module measuring grip strength, computer, large-size monitor, rack, and special motion sensing camera. It has 20 kinds of contents, and training can be performed in one of 5 stages according to the subject' s level. While the subject performs training, response time and scores are recorded. In this study, the 20 contents were divided into 5 groups, and the subjects had repeated training of them through 16 times for 8 weeks.

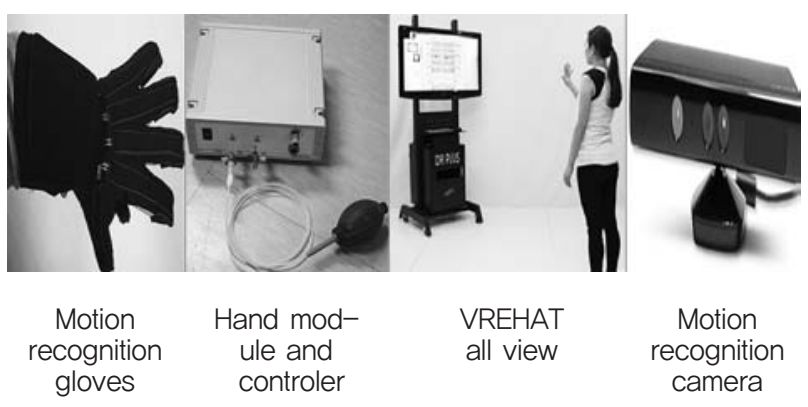

Fig. 1. virtual reality cognitive rehabilitation program

Table 1. Types of cognitive rehabilitation training program

\begin{tabular}{cc}
\hline times & contents \\
\hline 1 & window clear, \\
5 & lighting off(hand1), \\
9 & ball break out(leg), \\
13 & penalty kick, ADL kitchen task \\
2 & dot clear, \\
6 & lighting off(hand2), crosswalk \\
10 & ADL bathroom task \\
14 & lighting off(foot1), \\
3 & ball catch, animal match, \\
7 & Shooting, ADL room task \\
11 & ball break out(arm), Stairs, hand \\
15 & training, ADL work task \\
4 & lighting off(foot2), \\
\hline 16 &
\end{tabular}




\section{MEASUREMENT}

\section{Cognitive function test}

Cognitive function was measured using the Korean Mini-Mental Status Examination(K-MMSE) developed by Kwon Yong-cheol and Park Jong-han(1989) (8). This test can assess the subjects' intellectual status easily within a short time of about 5-10 minutes, and can estimate not only intellectual disabilities but also the level of functional development(9).

\section{Visual perception test}

The Motor-Free Visual Perception Test(MVPT), a test for assessing the functions of visual perception without including motor ability, is a standardized tool showing high reliability and validity in evaluating the general visual perception ability of both children and adults. This test is useful, taking a shorter time than other tools(10). MVPT consists of 36 questions in 5 areas, which are visual discrimination, figure-ground, visual memory, visual closure, and spatial relation, and each question is answered on a 1point scale. The score of the test indicates the subject's age of visual perception(11).

\section{Balance ability}

The subjects' functional performance of balance was measured using the Berg Balance Scale(BBS), the reliability and validity of which have been approved(12). This scale contains a total of 14 items on a $0 \sim 4$ point scale. The possible highest score is 56 , and a score between 41 51 indicates a low fall rate, between 21 40 a middle fall rate, and 0 20 a high fall rate.

\section{Lower limb strength(sit-to-stand)}

Lower limb strength was measured by counting the number of times that the subject, with his arms crossing each other on the chest, sat on a chair for 30 seconds and stood completely.

\section{Depression}

The Short-form Geriatric Depression Scale(GDS$\mathrm{K}$ ), which was developed originally by Yesavage et al. (1983) and adapted by Jeong In-gwa et al.(1997) for Korean people, measures simply by asking yes-no questions, and grasps the general phenomenon of depression by reflecting various aspects including intellectual, emotional, cognitive, physical and social aspects. A score between 14 18 indicates suspected or minor depression, between 19 21 moderate depression, and 22 or higher severe depression(13, 14).

\section{Data Analysis}

Data were analyzed using SPSS Version 22.0. Descriptive statistics and $\mathrm{x}^{2}$-test were used for subjects' general characteristics, and Mann-Whitney U test was used to test homogeneity between the groups. Comparison between before and after the training was made through Wilcoxon rank sum test, and effect test between the two groups was made through ANCOVA.

\section{RESULTS}

\section{Subjects' general characteristics}

According to the results of homogeneity test on the general characteristics of the experimental group and the control group, there was no statistically significant difference(p $>$.05)(Table2).

In the results of homogeneity test between the experimental group and the control group for dependent variables before participating in cognitive rehabilitation training, no statistically significant difference was observed between the two groups, and this suggests that the two groups had homogeneous characteristics(p〉.05)(Table3).

Table 2. Homogeneity Test for General Characteristics

\begin{tabular}{cccc}
\hline Variable & $\begin{array}{c}\text { Training Group } \\
(\mathrm{n}=15) \mathrm{N}(\%)\end{array}$ & $\begin{array}{c}\text { Control Group } \\
(\mathrm{n}=15) \mathrm{N}(\%)\end{array}$ & $\mathrm{p}$ \\
\hline Gender & & & \\
Male & $5(33.3)$ & $3(20.0)$ & .409 \\
Famale & $10(66.7)$ & $12(80.0)$ & \\
Age(years) & & & \\
$65-69$ & $1(6.7)$ & $1(6.7)$ & \\
$70-74$ & $1(6.7)$ & $4(26.7)$ & \\
$75-79$ & $4(26.7)$ & $6(40.0)$ & .290 \\
$80-84$ & $7(46.7)$ & $2(13.3)$ & \\
$>85$ & $2(13.3)$ & $2(13.3)$ & \\
Regular exercise & & & \\
No & $5(33.3)$ & $6(40.0)$ & .705 \\
Yes & $10(66.7)$ & $9(60.0)$ & \\
\hline
\end{tabular}


Table 3. Homogeneity Test for Dependent Variables

\begin{tabular}{cccc}
\hline Variable & $\begin{array}{c}\text { Training } \\
\text { Group(n=15) } \\
M \pm S D\end{array}$ & $\begin{array}{c}\text { Control } \\
\text { Group(n=15) } \\
M \pm S D\end{array}$ & p1) \\
\hline p1)K-MMSE & $16.40 \pm 5.64$ & $16.60 \pm 4.61$ & 1.000 \\
MVPT & $18.00 \pm 6.49$ & $18.40 \pm 6.98$ & .902 \\
GDS-K & $9.53 \pm 5.04$ & $10.33 . \pm 3.97$ & .539 \\
BBS & $49.80 \pm 3.64$ & $46.07 \pm 6.17$ & .067 \\
LLS & $12.2 . \pm 4.73$ & $10.40 \pm 4.82$ & .285 \\
\hline
\end{tabular}

K-MMSE(Korean Mini-Mental Status Examination)

MVPT(Motor-Free Visual Perception Test)

GDS-K(Short-form Geriatric Depression Scale)

BBS(Berg Balance Scale)

LLS(Lower Limb Strength)

\section{Comparison of the mean cognitive function}

In the result of comparing the groups on the mean difference of cognitive function between before and after the intervention, the posttest score was $18.87 \pm$ 5.41 in the experimental group and $16.27 \pm 4.55$ in the control group, so it was higher in the experimental group and the experimental group showed a statistically significant result but the control group did not. A statistically significant difference was observed between the two groups in effect test after controlling the pretest score of cognitive function as a covariate(p<.05)(Table 4).

\section{Comparison of the mean visual perception}

In the result of comparing the groups on the mean difference of visual perception between before and after the intervention, the posttest score was 20.00 5.30 in the experimental group and $17.80 \pm 6.72$ in the control group, so it was higher in the experimental group and the experimental group showed a statistically significant result but the control group did not. A statistically significant difference was observed between the two groups in effect test after controlling the MVPT pretest score of visual perception as a covariate $(\mathrm{p}<.05)$ (Table 5$)$.

\section{Comparison of the mean balance ability}

In the result of comparing the groups on the mean difference of balance between before and after the intervention, the posttest score was $50.93 \pm 2.73$ in the experimental group and $43.00 \pm 8.17$ in the control group, so it was higher in the experimental group and both the experimental group and the control group showed a statistically significant result. A statistically significant difference was observed between the two groups in effect test after controlling the pretest score of BBS as a covariate( $<$ <.05) (Table 6).

\section{Comparison of the mean lower limb strength}

In the result of comparing the groups on the mean difference of lower limb strength between before and after the intervention, the posttest score was 17.27 \pm 5.02 in the experimental group and $10.47 \pm 4.96$ in the control group, so it was higher in the experimental group and the experimental group showed a statistically significant result but the control group did not. A statistically significant difference was observed between the two groups in effect test after controlling the pretest score of lower limb strength as a covariate( $\mathrm{p}<.05)($ Table 7$)$.

\section{Comparison of the mean depression}

In the result of comparing the groups on the mean difference of depression between before and after the intervention, the posttest score was 5.53 \pm 3.62 in the experimental group and $12.00 \pm 5.47$ in the control group, so it was higher in the experimental group and the experimental group showed a statistically significant result but the control group did not. A statistically significant difference was observed between the two groups in effect test after controlling the pretest score of depression as a covariate (p<.05)(Table 8).

Table 4. The Comparison of cognitive function between Groups

\begin{tabular}{cccccc}
\hline Group & PreM \pm SD & PostM $\pm S D$ & DifferenceM $\pm S D$ & $p^{11}$ & $p^{2)}$ \\
\hline Training Group & $16.40 \pm 5.64$ & $18.87 \pm 5.41$ & $-2.46 \pm 2.74$ & .007 & .006 \\
Control Group & $16.60 \pm 4.61$ & $16.27 \pm 4.55$ & $0.33 \pm 2.49$ & .573 & \\
\hline
\end{tabular}

1) $P$ by Wilcoxon rank sum test

2) $P$ by ANCOVA(analysis of covariance) 
Table 5. The Comparison of Visual Perception between Groups

\begin{tabular}{cccccc}
\hline Group & PreM \pm SD & PostM $\pm S D$ & DifferenceM $\pm S D$ & $\mathrm{p}^{1)}$ & $\mathrm{p}^{2)}$ \\
\hline Training Group & $18.00 \pm 6.49$ & $20.00 \pm 5.30$ & $-2.00 \pm 2.44$ & .009 & .004 \\
Control Group & $18.20 \pm 7.01$ & $17.80 \pm 6.72$ & $0.40 \pm 2.84$ & .364 & \\
\hline
\end{tabular}

1) $P$ by Wilcoxon rank sum test

2) $P$ by ANCOVA(analysis of covariance)

Table 6. The Comparison of Balance between groups

\begin{tabular}{cccccc}
\hline Group & PreM \pm SD & PostM $\pm S D$ & DifferenceM $\pm S D$ & $p^{1)}$ & $p^{2)}$ \\
\hline Training Group & $49.80 \pm 3.64$ & $50.93 \pm 2.73$ & $-1.13 \pm 1.68$ & .024 & .000 \\
Control Group & $45.13 \pm 7.95$ & $43.00 \pm 8.17$ & $2.13 \pm 2.35$ & .006 & \\
\hline
\end{tabular}

1) $P$ by Wilcoxon rank sum test

2) $P$ by ANCOVA(analysis of covariance)

Table 7. The Comparison of Lower Limb Strength between group

\begin{tabular}{cccccc}
\hline Group & PreM \pm SD & PostM $\pm S D$ & DifferenceM $\pm S D$ & $p^{1)}$ & $p^{2)}$ \\
\hline Training Group & $12.20 \pm 4.73$ & $17.27 \pm 5.02$ & $-5.067 \pm 3.30$ & .001 & .000 \\
Control Group & $10.40 \pm 4.82$ & $10.47 \pm 4.96$ & $-.067 \pm 4.41$ & .766 & \\
\hline
\end{tabular}

1) $P$ by Wilcoxon rank sum test

2) $P$ by ANCOVA(analysis of covariance)

Table 8. The Comparison of Depression between Groups

\begin{tabular}{cccccc}
\hline Group & PreM $\pm S D$ & PostM $\pm S D$ & DifferenceM $\pm S D$ & $\mathrm{p}^{11}$ & $\mathrm{p}^{2)}$ \\
\hline Training Group & $9.53 \pm 5.04$ & $5.53 \pm 3.62$ & $4.00 \pm 4.08$ & .005 & .000 \\
Control Group & $10.33 \pm 3.97$ & $12.00 \pm 5.47$ & $-1.66 \pm 4.96$ & .183 & \\
\hline
\end{tabular}

1) $P$ by Wilcoxon rank sum test

2) P by ANCOVA(analysis of covariance)

\section{DISSCUSSION}

Dementia causes difficulties in normal life by damaging cognitive functions, and it shows gradual exacerbation and brings disabilities in activities of daily living, memory, and speech. Cognitive ability is essential to physical activities, and active physical activities maintain or improve cognitive functions (15). Accordingly, exercise and physical activities are indispensable to dementia patients(16). In the metaanalysis of 85 theses on the effects of exercise training in elders with cognitive damage and dementia, Heyn et al.(2004) reported that when physical training was applied to dementia patients it had a positive effect on their physical function, cardiovascular function, muscle strength, flexibility, cognition, activities of daily living, and behavior capability(17). In this study, we applied a virtual reality program consisting of exercise cognitive training tasks to the training group, and observed a significant difference in its MMSE score compared to that of the control group, suggesting the enhancement of the dementia elders' cognitive function. This result was consistent with the report of Kim(2002) that a virtual reality program for training elders' cognitive functions had a positive effect on the functions(18). While the virtual reality program in Kim(2002) research consisted of 16 tasks mainly for cognitive functions, however, the virtual reality program applied in this 
study contained 20 tasks for exercise and cognitive training and, as a result, it showed a higher effect than the program containing only tasks for cognitive functions.

For elders with deteriorated visual motor ability, their poor vision had an adverse effect on their acquisition and interpretation of information, which, in turn, brings changes in their behavioral characteristics and acts(19). In this study, the mean score of visual perception after the intervention showed an increase in the training group, but a slight decrease in the control group. There was a significant difference between the two groups. Witt et al.(1988) thought perception as an intermediate stage between sensation and cognition(20). Recent perception theories argued that visual perception affects and is affected by cognition(20, 21). In this study as well, improvement in cognitive functions was observed and this suggests an effect on visual perception. In addition, the subjects carry out tasks through interaction between the real self and the self displayed on the screen in the virtual reality program, and visual feedback is believed to have a positive effect on their visual perception ability, in particular, their eyehand coordination ability(22). Because they had to wear gloves for performing delicate cognition tasks and had to repeat a task if they made a mistake, moreover, it is believed that visual feedback had an effect.

In the results of measuring BBS (balance ability) in virtual reality cognitive rehabilitation training, the control group showed a difference of $2.13 \pm 2.35$ between before and after the intervention, and their balance ability decreased after the intervention compared to that before. The training group showed a difference of $-1.13 \pm 1.68$ between before and after the intervention, suggesting that their balance ability increased. In his study on the effect of a virtual reality game on dementia patients' improvement in cognition and balance, Lee Jin-ho(2010) reported that the BBS score was statistically significantly higher in the experimental group than in the control group(23). Shin Won-seop \& Lee Seok-min(2009) measured BBS in order to examine the effect of rehabilitation exercise using virtual reality on brain injury patients' functional recovery, and according to the results, BBS increased by 5.3 from 45.4 to 50.7 in the group that used virtual reality, showing improvement in balance ability, but it was similar to or even lower than that before exercise in the control group, and this is consistent with the result of this study(24). In the study by Walker et al.(2000) as well, the application of rehabilitation exercise using visual feedback increased the BBS score by 10.7, proving the effect of feedback on balance ability. Based on this result, it is believed that while performing dynamic tasks in virtual reality, the subjects see their own balance state and movements and control balance using the visual feedback and this improves their balance control ability(25).

When lower limb strength was measured in this study, there was a significant difference between the two groups. This is probably because the subjects had to keep standing for 20-30 minutes while carrying out tasks in the intervention program, they had aerobic exercise and this improved not only their cognition but also their lower limb strength. Thomas \& Hageman(2002) applied resistance training, which consisted of lower limb exercise for strengthening lower limb muscles and physical functions, to dementia patients for 6 weeks, and reported that muscle strength and in particular gait ability increased significantly and therefore the exercise was effective in decreasing the risk of fracture in dementia patients(26). What is more, Song Chang-ho et al.(2009) applied a virtual reality program using video games to elders and reported that the program was effective in improving their lower limb strength, balance and gait, supporting the results of this study (27).

As to the effect of the intervention program on depression, the difference between before and after the intervention was statistically significant in the training group but not in the control group and the result of effect test was also different between the two groups. These results show that the intervention program has an effect on depression. Among previous studies as well, Lee Sang-yeol et al.(2012) reported that virtual reality games applied to elders decreased their depression(28, 29). People's physical activities decrease with aging, and when a virtual reality game is used to elderly patients in such a situation they can enjoy the interaction as if playing a game rather than having a therapeutic process(30). Such a pleasure of interaction motivates the patients, and increases their participation in activities(31). Brach(2003) also reported that appropriate physical activities for female elders had positive effects on them such as refreshing their feeling and giving mental stability(32).

However, this study has a limitation in generalizing the results because the control group was not sampled at random from the same center, the period of the program was short, and the sample size was small. Thus, further research in various approaches is required in the future. 


\section{CONCLUSION}

This study purposed to examine how the application of a virtual reality cognitive rehabilitation training system to dementia elders affects their cognitive function, visual perception, depression, balance ability, and lower limb strength. Compared to the control group, the training group showed a significant difference between before and after the intervention in cognitive function, visual perception, depression, balance ability, and lower limb strength. In the control group, however, no significant difference was observed in cognitive function, visual perception, depression, and lower limb strength between before and after the intervention. Particularly in balance ability, the control group rather showed a decrease after the intervention. The difference between the experimental group and the control group was significant in all the measured items.

Thus, this study is considered meaningful in that it tested the potential of virtual reality cognitive rehabilitation training as a new cognitive rehabilitation program and proved the effectiveness of the virtual reality cognitive rehabilitation training system containing specialized cognitive rehabilitation contents.

\section{REFERENCES}

1. Ryu, HJ. The Comparison on SNSB Performanc among Normal Age Person, Mild Cognitive Impairment and Early Dementia. Graduate School of Rehabilitation Science Daegu University 2011.

2. Eschrich S, Munte T. F. \& Altenmuller E. O. Unforgettable film music. The role of emotion in episodic long-term memory for music. BMC Neuroscience 2008; 9(1): 48.

3. Weiss P. L, Rand D, Katz N \& Kizony R. Video capture virtual reality as a flexible and effective rehabilitation tool. J Kor neuroengineering and rehabilitation 2004; 1(1): 12.

4. Lott A, Bisson E, Lajoie Y, Mc Comas J \& Sveistrup H. The effect of two types of virtualreality on voluntary center of pressure displacement. Cyber psychol Behav 2003; 6: 477-485.

5. Kim JH, Kim CS. Effects of virtual reality program on standing balance in chronic stroke patients. J Kor Society of Physical Therapy 2005; 17(3): 351-367.
6. Jang JY, Yoon YS. A Study on Serious Game Design for Rehabilitation Training of Sroke Patients. The Korean Society for Computer Game 2008; 15: 151-159.

7. Kim JH, Oh MH, Lee JS, Ahn HS. The Effects of Training Using Virtual Reality Games on Stroke Patients' Functional Recovery. The Journal of Korean Society of Occupational Therapy 2011; 19(3): 101-114.

8. Gwon, YC, Park, JH. Elderly Korean version of Mini-Mental State Examination Standardization study(MMSE-K)-Part 2: division and iagnostic validity NeuroPsychiatry. 1989; 28(3): 125-135.

9. Han TR, Kim JH. Rehabilitation medicine. Koonja 2002.

10. Korean Association for Geriatric Psychiatry, Korean dementia assessment tests. hakjisa 2003.

11. Mercier L, Hebert R, Colarusso R, Hammill D. Motor-Free Visual Perception Test-vertical Manual. Califonia: Academic Therapy Publications 1997.

12. Berg K, Wood-Dauphinee S, Williams JI, Maki B. Measuring balance in the elderly: preliminary development of an instrument. Physiotherapy Canada 1989; 41: 304-311.

13. Yesavage JA, Brink TL, Rose TL, Lum O, Virginia H, Adey M, Leirer VO. Development and Validation of a Geriatric Depression. Screening Scale: A Preliminary Report. J. Psychiat. Res 1983; 17(1): 37-49.

14. Jeong IG, Gwak DI, Sin DG, Lee MS, Lee HS, Kim JY. Reliability of the Geriatric Depression Scale, Validity. Neuropsychiatric Medicine. 1997; 36(1): 103-111.

15. Ryu KH, Oh KJ. Neuropsychological Differentiation Between Mild Alzheimers Disease and Subcortical Vascular Dementia. Korean Psychological Association. 2006; 25(4): 987-1010.

16. Im JH. A Review of literature on effects of regular exercise on physical and cognitive functions among demented elders. Sung Shin Woman's university 2007.

17. Hyen P, Abreu BC, Ottenbacher KJ. The effect of exercise training on elderly persons with cognitive impairment and dementia Arch Phys Med Rehabil 2004; 85(10): 1694-704.

18. Kim MY, Lee KS, Choi JS, Kim HB, Park CI. Effectiveness of Cognitive Training based on Virtual Reality for the Elderly. J Korean Acad Rehabil Med. 2005; 29(4): 424-433.

19. Choi MH, Kim HJ, An OH. A Literature Study on the Elderly's Visual- Perception : Focusing on the Domestic Research. Journal of Korean Institute 
of Illuminating and Electrical Installation Engineers 2009; 23(1): 1-8.

20. Lee HS. Evaluation of the Visual Perception Ability and it's factors on MVPT-3 test in the Elderly Population. Graduate School Chonnam National University 2010.

21. Colarusso RP, Hammill DD. Motor-Free Visual Perception Test-3 Manual. California Academic therapy publications 2003.

22. Shin JW. Effects of Virtual Reality Training Program on Eye-Hand Coordination in Children with Cerebral Palsy. Graduate School of Rehabilitation Science Daegu University 2012.

23. Lee Jh. Influence of virtual reality games on cognition, balance and function in dementia patients. Graduate School of Public Health Nambu University 2010.

24. Shin WS, Lee SM. Effects of Rehabilitation Exercise Using Virtual Reality on Functional Recovery in the Persons with Stroke. Journal of Special Education \& Rehabilitation Science 2005; 48(3): 49-64.

25. Walker C, Brouwer BJ, Culham EG. Use of visual feedback in retiraining balance following acute stroke. Physical therapy 2000; 80(9): 886-895.

26. Thomas VS, Hageman PA. A preliminary study on there liability of physical performance measures in older day-care center clients with dementia. Int Psychogeriatr 2002; 14(1): 17-23.

27. Song CH, Shin WS, Lee, KJ, Lee SW. The effect of a virtual reality-based exercise program using a video game on the muscle strength, balance and gait abilities in the elderly. Journal of the Korean Gerontological Society 2009; (4): 1261-1275.

28. Lee SY, Lee SK, Kim YH, Chae JB. The Effects of 4 Weeks Training using Virtual Reality Game on Balance and Gait Ability, Depression of Elderly People. J Korea Proprioceptive Neuromuscular Facilitation Assocition. 2012; 10(2): 41-46.

29. Shin KE. Effect of Virtual Reality Game on Old Patient's Depression, Relationship and Life Satisfaction. Graduate School of Dong Shin University 2014.

30. Holden MK, Dyar T. Virtual environment training: A new tool for neurorehabilitation. Neurology Report 2002; 26: 62-74.

31. Kwon JS, Yang NY. Feasibility of Virtual Reality for Enhancement of Upper Extremity Function Post Stroke. Therapeutic Science for Neuro rehabilitation 2012; 1(2): 35-40.

32. Brach. Physical activity and functional status incommunity-dwelling older women: A 14-year peospective study. Archives of internal Medicine 2003; 163(21): 2565-2571. 